\title{
Multi-alternative control of large systems
}

\author{
Semen Podvalny ${ }^{*}$ and Eugeny Vasiljev \\ Voronezh State Technical University, 394026 Voronezh, Russia
}

\begin{abstract}
The article deals with analysis of evolutionary concept of control of large systems - multialternativeness. Based on the biocybernetical approach to the task we are revealing information components of this concept, which is formulated in the following 3-M principles: multilevel structure is implementing in complex systems as the property of homeostasis; multi-version of algorithms and separation of control functions for the adaptive systems control; modularity as guarantee for this diversity.
\end{abstract}

\section{Definition of a problem}

The solution of the problem of large systems control within the robust-adaptive algorithms meets the difficulties of physical implementation of these algorithms arising due to big size of the systems themselves, their internal interrelations, nonstationarity of their parameters and dynamic uncertainty.

At the same time, a cybernetic approach to the analysis of this task indicates the condition that in biological systems the contrary phenomenon is observed: i.e., the growth of biosystems complexity causes the increase of their ability to the balanced development under the conditions of unpredictable change of the external environment and pressure of natural selection.

A specified property of «non-complicating simplicity» of live organisms and systems gives the grounds to assume that their activity is the cornerstone of certain evolutionary principles and their reproduction in anthropogenous systems (i.e., generated by human intellect) gives the way to realize a truly degree of stability and balance in them in the form of a direct analog of a biological homeostasis [1].

In this work the following principles are offered [2]:

- the principle of variety and division of functions;

- the principle of a multilevel control structure;

- the principle of a modularity,

all of them forming the general concept of multialternative control (from Latin alternare - to alternate) $[3,4]$.

Actual trends of usage of the of multi-alternative control principles, their initial biological content and the particular examples of their technical realization are shown below.

\section{Actuality of multi-alternative control}

Multi-choice control was actively used as early as 1957 by A.M. Lyotov to solve the problems of automatic control of dynamic objects, who offered to change the feedback sign in the regulator, i.e. to change its structure, choosing one of two alternatives depending on the current state of an object. Further studies of this problem have led to the development of systems with variable structure [5]. Presently, this trend developed into the theory of hybrid (switched) control systems combining the properties of continuous and discrete systems [6-8]. There are the potentialities of the formation of complex nonlinear control rules in the switched systems, which change depending on the changing external factors of the environment.

The principle of variety is included in the mandatory recommendation of the International Power Commission to develop the emergency protection systems for nuclear power plants. Such systems were identified as diversity system. The most commonly used forms of diversity in the protection systems are: technical, functional, parametrical, software and design forms. An example of technical diversity is the realization of the active protection which transforms the power unit to the state of safety in the presence of an electric power source, and the passive, nonvolatile protective option providing this safety only by force of gravity.

Due to the expansion of microprocessor control and the unprovability of nonfailure software operation of the automation equipment, the actuality of implementation of the diversity principle is the realization of the software programmable functions using the non-programmable means. Presently, the principle of diversity is being developed as the main method to increase the software reliability.

In the spaceships automatic control systems the latest tendency is to avoid the hardware reservation and the transition to reconfigured systems providing the flight safety with the artificially formed structural redundancy, i.e., the variety of control algorithms.

Corresponding author: vgtu-aits@yandex.ru 


\section{Biological sources of multi- alternativity}

\subsection{Principle of variety and division of functions}

The most important prerequisite of existence and evolution of all biological systems on Earth under the influence of the environment is the mandatory variety of its taxons due to which in the situations when the change of habitat becomes adverse for the existence of an arbitrary element of the system its function is taken by the other one (or many) biological groups that are capable of restoring the broken balance in the system, e.g., the continuity of a ffiood chain.

At the level of individual organisms the diversity of adaptive mechanisms is exposed in the form of differentiation and division of functions of their cells and organs for use in various environments within the natural habitat. The nature chose do develop the diversity, but not combination of functions in «universal» organs and systems of organisms. Animals vision is the example of the result of evolution which led to the formation of colorsensitive vision, but with low photosensitivity of the visual receptors functioning in the daylight and the highly sensitive receptors that do not sense color and function when the luminance is low.

Another more amazing example of the diversity principle and the division of functions is the presence of the so-called stem cells in the highest mammal forms which initially were not functionally focused, however, being capable to get narrow functional specialization depending on the current requirements of an organism.

This diversity provides stability of existence of the biological systems and their effective consumption of energy from both the external environment and internal resources.

\subsection{Principle of multilevel control structure}

In biosystems the multi-alternativity is implemented not only in the variety of the live world, but also as a multilevel structure, the hierarchy of this variety. Hierarchy of a structure is the basis of the most important property of all live systems, i.e. the principle and phenomenon of homeostasis [1] which activates higher levels of protection or reaction of an organism in the process of exhaustion of lower protection levels.

This property is implemented generally at the expense of a control system with two or more hierarchical levels one of which continuously supports the preset condition of an organism under the lowscale (insignificant) environmental changes, whereas the other levels react only to critical deviations of these conditions and as soon as these deviations manifest themselves, these protection levels start the qualitatively new mechanisms of essential reorganization of an organism, particularly, the spasmodic, uneven mechanism generating the alternative control structures, as shown in Figure 1.

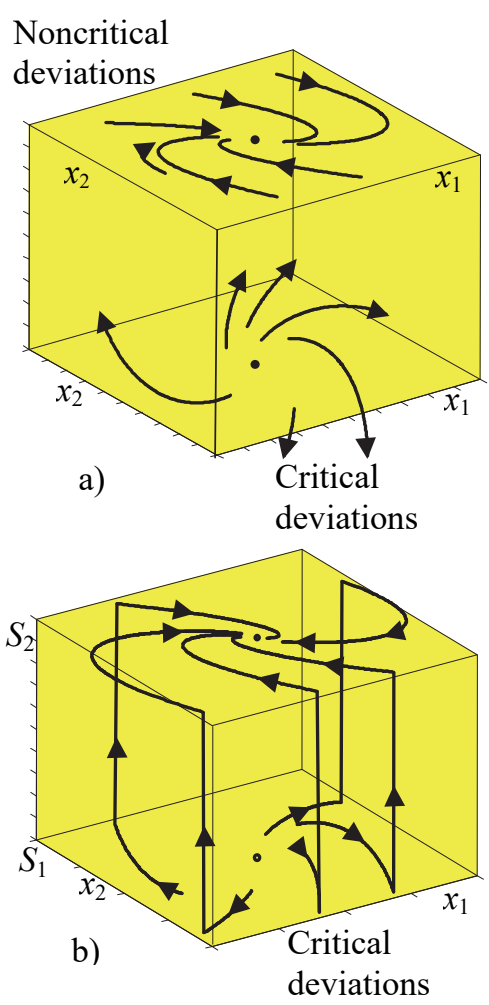

Fig. 1. Principle of multi-alternative control: a) parametrical level; b) structural level - the transition from structure $S_{1}$ to structure $S_{2}$

W. Ashby [1], distinguished five (sic!) independent mechanisms of stabilization of glucose levels in the blood of a human.

The hierarchy of control forms, as a result of accumulation, the expanded set of tools, the multilayered protective «belt» which protects the system from unpredictable changes of the environment.

\subsection{Principle of a control structure modularity}

The source of diversity is the principle of a modularity which manifests itself in the formation of new live structures from the already available and rather small number of biologically stable modules [9]. The modularity of a structure is the major prerequisite of the «non-complicating simplicity» of the live world.

In particular, the known variety of proteins is realized as combinations of 20 basic amino acids the placement of which in protein is encoded in the genetic code in the form of a chain of triplets using only four nucleotides: adenine, guanine, thymine, cytosine. This set of blocks provides all proteinaceous variety of fauna: the chain comprising only 100 triplets can form 20100 variants of protein; the actual size of such chains may be several thousand triplets and even tens of thousand triplets. Discussing the development mechanisms, E. M. Galimov [10] notes that «the most economical way of the formation of a low-entropy product is the 
combination of already available low-entropy structures, and the evolution, as we should understand it should comprise not only and, moreover, not all possible small changes but the spasmodic changes caused by the new combinations of existing structures», thus emphasizing the combinatory nature of evolution. The discussed multi-alternative approach to the control in complex systems is formulated by W.Ashby as the information principle of the essential variety: «Variety can destroy variety».

The list of biological examples of multi-alternative control shown below brings us to the conclusion that complex biological systems have reached the high level of adaptive interaction with the environment by means of selective specialization of this interaction on the basis of: a set of considerably simple mechanisms of the division of functions; modularity and hierarchy of structure, thus they avoid the restrictions imposed by the growing functional complexity of living organisms. These adaptive mechanisms are united with the universal concept of multi-alternativity.

\section{Examples of realization of multi- alternative control}

\subsection{Process of counterflow heat exchange}

Let's review an example of realization of the multialternativity principles in the control system of twocircuit counterflow heat exchange process, which is shown schematically in Figure 2.

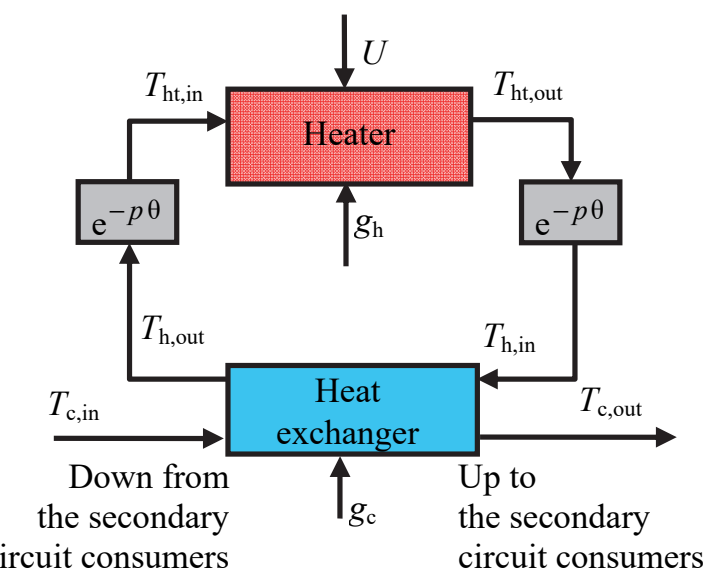

Fig. 2. Counterflow heat exchange process.

Here: $T_{\mathrm{c}, \text { in }}, T_{\mathrm{c}, \text { out }}$ are the temperatures of the "cold" secondary circuit heat-transfer agent at the input and output of the heat exchanger, respectively; $T_{\mathrm{h}, \text { in }}, T_{\mathrm{h} \text {,out }}$ are the temperatures температуры of the "hot" primary circuit heat-transfer agent at the input and output of the heat exchanger, respectively; $T_{\mathrm{ht}, \text { in }}, T_{\mathrm{ht}, \mathrm{out}}-$ are the temperatures circuit heat-transfer agent at the input and output of heater; $\theta$ is the time required for the heat exchanger to pass the distance between the heat exchanger and the heater; $\mathrm{e}^{-p \theta}$ is the transfer function of the delay element; $g_{\mathrm{c}}, g_{\mathrm{h}}$ are the mass flow rates of the heat-transfer agent (per second) in the secondary circuit (the consumers' circuit) and the heater circuit, respectively; $U$ is the temperature of the heater's external surface. Proceeding from the aprioristic uncertainty and the nonstationarity of the parameters of an object, the controllers may be synthesized in the class of the fuzzy control, having the potential to form independent individual control rules for various conditions of a controlled object. This opportunity corresponds nicely to the declared multi-alternativity principles. According to the multilevel principle we shall construct the block diagram of an automatic control system with five control levels in each channel of impact $g_{\mathrm{h}}$ and $U$, as shown in Figure 3.

Basing on of the current value of controlled temperature $T_{\mathrm{ht} \text {,out }}$ the linguistic variable $\widetilde{T}_{\mathrm{ht} \text {,out }}=\gamma$ is formed where $\gamma$ contains the fuzzy variables $\gamma: \gamma=\left\{\gamma_{1}\right.$, $\left.\gamma_{2}, \gamma_{3}, \gamma_{4}, \gamma_{5}\right\}$, using the measures of comparison with the required average value of $T_{\mathrm{ht}, \text { out,r }}$.

The increment $\Delta g_{\mathrm{h}}$ of a mass flow rate $g_{\mathrm{h}}$ is put in compliance with the linguistic variable $\alpha$ : $\alpha=\left\{\alpha_{1}, \alpha_{2}\right.$, $\left.\alpha_{3}, \alpha_{4}, \alpha_{5}\right\}$ with the values using the extent of a change $\alpha$ in comparison with its current level. For fuzzy variables $\alpha_{1}, \ldots, \alpha_{5}$ separate control channels are added, the contribution of each such channel into the resulting fuzzy impact $\beta$ developed by the controller shall be defined by a measure of proximity of a current state $\gamma$ to the required state $\gamma_{r}$ :

$$
\gamma_{\mathrm{r}}=\left[\mu_{\gamma 1}\left(T_{\mathrm{ht}, \text { out }, \mathrm{r}}\right), \mu_{\gamma 2}\left(T_{\mathrm{ht}, \text { out }, \mathrm{r}}\right), \ldots, \mu_{\gamma 5}\left(T_{\mathrm{ht}, \text { out }, \mathrm{r}}\right)\right]
$$

Using the Cartesian product of $\gamma$ and $\gamma_{\mathrm{r}}$, we obtain the transition matrix of $\mathrm{M}\left(\gamma \times \gamma_{\mathrm{r}}, \beta\right)$ from the current state $\gamma$ to the required state $\gamma_{\mathrm{r}}$ under the influence of the sought for control $\beta$ :

$$
\mathrm{M}\left(\gamma \times \gamma_{\mathrm{r}}, \beta\right)=\gamma \times \gamma_{\mathrm{r}}
$$

For the definition of the general control $\beta$ for each of $n$ channels of the system the transition matrices of $\mathrm{M}\left(\gamma_{i j} \times \gamma, \alpha_{s}\right), i=1 \ldots n ; j=1 \ldots n ; s=1 \ldots n ; n=5$ are formed and the values of their elements characterize the possibility of a transition of an object from the condition $\gamma_{i}$ to the condition $\gamma_{j}$ under the influence of control $\alpha_{s}$. As a measure of proximity of the required control $\beta$ to the known controls $\alpha_{1}, \ldots, \alpha_{5}$ the extent of inclusion of a transition matrix $M\left(\gamma \times \gamma_{r}, \beta\right)$ in the corresponding matrices is applied to each channel:

$$
v_{s}=v\left(\beta, \alpha_{s}\right)=v\left[M\left(\gamma \times \gamma_{r}, \beta\right), M\left(\gamma_{i} \times \gamma_{j}, \alpha_{s}\right)\right]
$$

The received values of the proximity measures of $v_{s}$ are used for logical conclusion:

$$
\mu_{s}\left(\Delta \mathrm{g}_{\mathrm{h}}\right)=\&_{\Delta \mathrm{gh}}\left(v_{s}, \mu_{\alpha s}\left(\Delta \mathrm{g}_{\mathrm{h}}\right)\right)
$$

the result of which $\mu_{s}\left(\Delta g_{h}\right)$ are the functions of accessory $\mu_{\alpha s}\left(\Delta g_{h}\right)$ truncated at the $\nu_{s}$ level. Control $\beta$ can be calculated using the operation of association applied to all fuzzy subsets $\mu_{\mathrm{s}}\left(\Delta \mathrm{g}_{\mathrm{h}}\right)$ : 


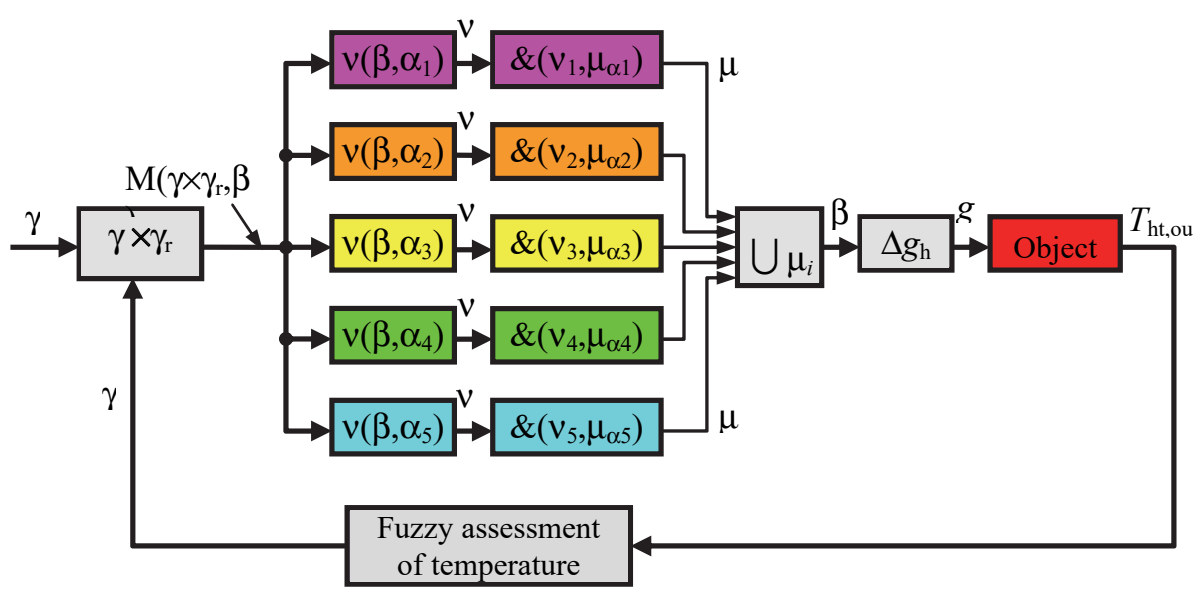

Fig. 3. Block diagram of a 5-level $g_{\mathrm{h}}$-controlled control channel.
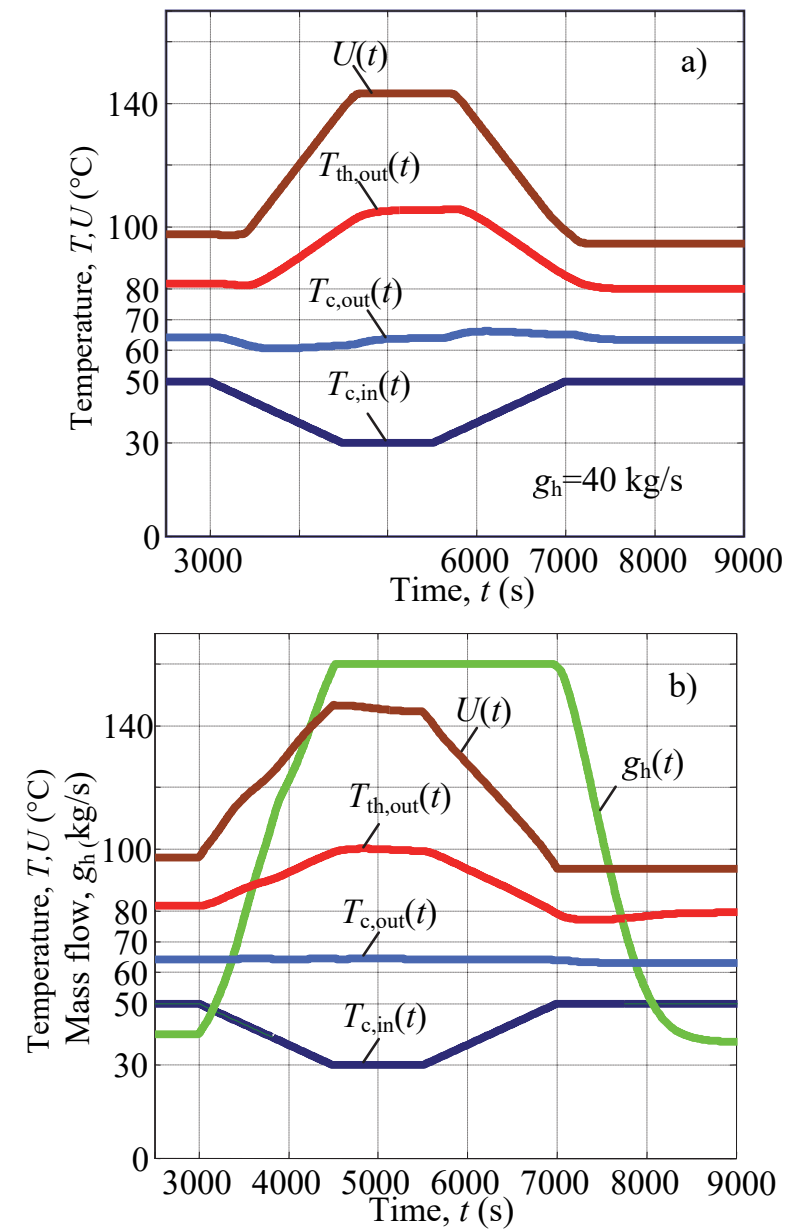

Fig. 4. Transition processes in the heat exchange control system with: (a) one control channel, and (b) three control channels with five control levels in each channel.

$$
\left.\beta=\left\{\mu_{s}\left(\Delta \mathrm{g}_{\mathrm{h}}\right)\right\}=\left\{\cup_{\Delta \mathrm{gh}} \mu_{\alpha s}\left(\Delta \mathrm{g}_{\mathrm{h}}\right)\right)\right\} .
$$

Procedures (1)-(5) define the multi-alternative algorithm utilizing the $g_{\mathrm{h}}$ control channel. The control channel is constructed similarly utilizing the heater temperature $U$.

The content of this algorithm has clear physical meaning: at a shortage of resources of low level control the higher hierarchical level of the system is started providing the required quality of control as a result. The modular structure of each level of a system allows to create the control $\beta$ in the form of multidimensional function $\beta\left(v_{1}, v_{2}, v_{3}, v_{4}, v_{5}\right)$, i.e., generally, in the form of a $n$-dimensional vector, providing high flexibility of control in the full range of current object states. In Figure 4a the result of work of control system with a single control channel by $g_{\mathrm{h}}$ is shown. The system keeps the value $T_{\mathrm{c}, \text { out }}$ in the preset limits $60 \ldots 70^{\circ} \mathrm{C}$, however the value of 
temperature $T_{\mathrm{ht}, \text { out, }}$ of the heat-transfer agent at the output of the heater exceeds the critical value $100^{\circ} \mathrm{C}$.

To eliminate this shortcoming according to the diversity principle the two additional control channels are added to the system, one of them utilizing the heater temperature $U$, and the other channel using the disturbance input $T_{\mathrm{c}, \text { in }}$. The quality of $T_{\mathrm{c} \text {,out }}$ and $T_{\mathrm{th}, \text { out }}$ control in the final multi-alternative system is improved considerably as confirmed by Figure $4 \mathrm{~b}$.

\subsection{The space station power supply system}

The space station power supply system includes three levels of subsystems, shown in Figure 5a: solar batteries (SB) subsystem; external of high voltage source (ES) subsystem; electrochemical storage batteries (EB) subsystem. Interaction principle of these subsystems results in the hierarchical structure in which the transfer of power supply function to each subsequent level occurs only in the condition of completely exhausted power resources of the previous level.
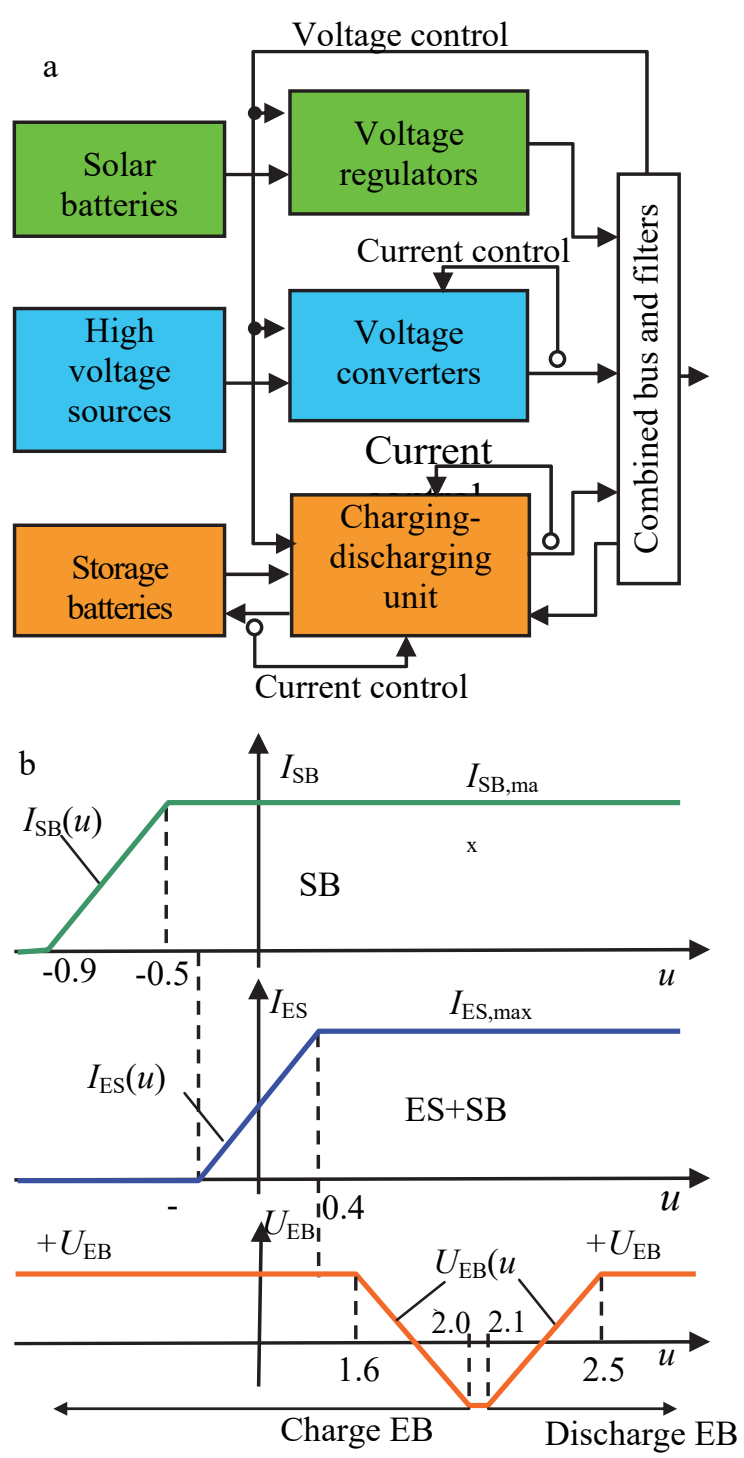

Fig. 5. An example of the multi-alternativity principles in the structure and operating modes of space station power supply system.
In Figure $5 b$ it is shown that at the shift of the operating signal $u$ outside the control range $[-0.9 ;-0.5]$ the solar batteries subsystem provides its full current $I_{\mathrm{SB} \text { max }}$, to the load thus starting the external power source subsystem (control range [-0.4; 0.4]). With the solar battery completely loaded and selection of an external power source $\left(I_{\mathrm{SB}}=I_{\mathrm{SB}, \max }\right.$ and $\left.I_{\mathrm{ES}}=I_{\mathrm{ES} \text {,max }}\right)$ power supply from storage batteries is started. Similar transfer of control is carried out within each subsystem which contains a large number of identical modules. The modularity and hierarchy of a structure exclude any possibility of cascade (technologically conditional) increase of failures in the system whereas at any current in the load active control is provided for just a part of power supplied to power consumers corresponding to one single module of this either one or some other subsystem. All other modules are in the uncontrolled mode, some generates maximum current, and the others that are not required at the moment are disconnected.

As a result achieved in this «non-complicating simplicity» system, there is a possibility to unify the control circuits and the development of modular subsystems from interchangeable modules thus resulting in the reliable functioning of the whole power supply system in the wide range of deviations of its parameters, loads and at failures of various individual modules.

These examples adequately demonstrate that multi-alternative control of complex systems provides high quality of their functioning not just as a result of formation of the general algorithm of either robust or adaptive control, but by means of independent, autonomous synthesis of the corresponding hierarchical subsystems.

\section{Conclusions}

The solution of control tasks of complex systems by means of synthesis of uniform universal robust-adaptive algorithms is characterized by the accruing contradiction between the theoretical results and the extent of their realization achieved in this direction in practice.

Focusing on the biological origins of the problem being considered shows that the variety of conditions of the environment in the course of evolution of live organisms have led to the development of their corresponding adaptive mechanism: the multialternativity of functioning.

The concept of multi-alternativity offered in this work as a way of understanding the mechanisms of evolution and adaptation in living organisms gives the opportunity of purposeful reproduction of these mechanisms into the control systems of complex objects. This concept determines by itself the «non-complicating simplicity» principle as a reply to the problems of large systems development. Examples of realization of this mechanism in the automatic control systems utilized for various purposes confirm its high efficiency.

The offered way of a solution of the problem of large systems control follows in the course of the quickly developing scientific direction, viz., the 
biological cybernetics, which studies along with the basic feedback principle, the deeper analogies of anthropogenous systems with evolutionary principles of the biological communities functioning, which determine their final existence and the development.

\section{References}

1. W.R. Ashby, Design for a brain (London: Chapman \& Hall, 1966)

2. S.L. Podvalny, E.M. Vasiljev, Autom. Remote Control, 8, 1471-1499 (2015)

3. S.L. Podvalny, E.M. Vasiljev, Autom. Remote Control, 2, 311-317 (2015)

4. S.L. Podvalny, E.M. Vasiljev, V.F. Barabanov, Autom. Remote Control, 10, 1886-1891 (2014)

5. S.V. Emelianov, S.K. Korovin, I. G. Mamedov, Variable-structure control systems: discrete and digital, (Inc. Boca Raton, FL, CRC Press, 1995)

6. D. Liberzon, Switching in systems and control, (Boston, MA, Birkhäuser, 2003)

7. S.N. Vassilyev, A.A. Kosov, Autom. Remote Control, 6, 1163-1183 (2011)

8. S.L. Podvalny, E.M. Vasiljev, International conference «Stability and control processes in memory of V.I. Zubov» (SCP), 120-122 (2015)

9. V.A. Krassilov, Theory of evolution: a new synthesis is needed, evolutionary research. Macroevolution, (Vladivostok, Dal'nevost. Nauchn. Tsentr, 1984)

10. E.M. Galimov, Phenomenon of life, between equilibrium and non-linearity. Origin and principles of evolution, (Moscow, Editorial URSS, 2006) 